Kansas State University Libraries

New Prairie Press

\title{
THE ANALYSIS OF THE ADDITIVE MIXED MODEL FOR CLASSES OF NON ORTHOGONAL DESIGNS
}

Christine A. Stidley

Follow this and additional works at: https://newprairiepress.org/agstatconference

Part of the Agriculture Commons, and the Applied Statistics Commons

\section{(c) (1) $\Theta($}

This work is licensed under a Creative Commons Attribution-Noncommercial-No Derivative Works 4.0 License.

\section{Recommended Citation}

Stidley, Christine A. (1989). "THE ANALYSIS OF THE ADDITIVE MIXED MODEL FOR CLASSES OF NON ORTHOGONAL DESIGNS," Conference on Applied Statistics in Agriculture. https://doi.org/10.4148/ 2475-7772.1466

This is brought to you for free and open access by the Conferences at New Prairie Press. It has been accepted for inclusion in Conference on Applied Statistics in Agriculture by an authorized administrator of New Prairie Press. For more information, please contact cads@k-state.edu. 
THE ANALYSIS OF THE ADDITIVE MIXED MODEL FOR

CLASSES OF NONORTHOGONAL DESIGNS

Christine A. Stidley

New Mexico Tumor Registry

Cancer Treatment and Research Center

University of New Mexico Medical Center

Albuquerque, NM 87131

\section{ABSTRACT}

Tests for fixed and random effects can be difficult to derive for nonorthogonal designs with mixed models. However, extensions of the intrablock and inter-block analyses of Balanced Incomplete Block Designs can often be obtained. Here we derive the extensions for the broad class of Group Divisible Designs. Decompositions of the design space are used to develop exact tests for fixed and random effects in the additive mixed model with random block effects. Conditions on the design which permit the standard use of the intra-block and inter-block test statistics are given. Important subclasses of Group Divisible Designs include Equireplicate Variance Balanced Block Designs and Group Divisible Partially Balanced Incomplete Block Designs with Two Associate Classes. These two subclasses are also examined. An example from the literature of an experiment on fruit trees is used to illustrate the methods.

Key words: Analysis of variance; Group Divisible Designs; Intra-block analysis; Inter-block analysis; Variance Balanced Block Designs.

\section{INTRODUCTION}

We consider the class of Group Divisible Designs (GDDs), which includes such designs as Group Divisible Partially Balanced Incomplete Block Designs with Two Associate Classes (GDPBIBDs), Equireplicate Variance Balanced Block Designs (EVBDs) and Balanced Incomplete Block Designs (BIBDs). While much attention has been given to the construction of these designs and subclasses (see, for example, Freeman (1976), Gupta and Jones (1983), Hedayat and Federer (1974), Kageyama (1981), Kageyama and Mohan (1985), Sinha (1987), and Tyagi (1979)), distribution theory has not been obtained for the general class of designs or for many of the subclasses.

First, we describe this class of designs. Then by using extensions of the intra-block and inter-block analyses for BIBDs, we derive exact distribution theory for tests for fixed and random effects for the additive mixed model with random block effects. Details of the derivations are given in the Appendix. An example is also given.

\section{DESCRIPTION OF GROUP DIVISIBLE DESIGNS}

Let $t$ be the number of treatment levels, $b$ the number of blocks, $n$ the number of observations, $k_{j}$ the size of the $j$ th block, $k=\left(k_{1}, \ldots, k_{b}\right)^{\prime}$ and $r_{0}=\left(r_{1}, \ldots, r_{t}\right)^{\prime}$, the vector of treatment replicates. Let $X_{A}$ and $X_{B}$ be the zero-one design matrices for treatments and blocks, respectively. 
Consider the $t \times t$ matrix $\Omega^{-1}$, which is given by

$$
\Omega^{-1}=R-N K^{-1} N^{\prime}+r_{0} r_{0}^{\prime} / n
$$

where $R$ and $K$ are diagonal matrices with diagonals of $r_{0}$ and $k$, respectively, and $N=X_{A}^{\prime} X_{B}$, the treatment-block incidence matrix. The matrix $\Omega^{-1}$ is important because of its role in the intra-block estimation of treatment effects (Tocher (1952)). Pearce (1963) uses this matrix to classify designs.

In Pearce's classification scheme a GDD is any design such that

$$
\Omega^{-1}=c_{1} I_{t}+c_{2} I_{m}^{\otimes} J_{s}+c_{3} J_{t} \text {, }
$$

where $I_{t}$ is the $t \times t$ identity matrix, $J_{t}$ is a $t \times t$ matrix of ones, $c_{1}$, $c_{2}$ and $c_{3}$ are scalars and $\otimes$ denotes direct product. From (2.2) it can be seen that the $t$ treatments are divided into $m$ groups of $s$ treatments. Treatments are thus nested within groups. Let $X_{M}$ be the zero-one design matrix for groups. Differences in treatment effects are estimated with the same variance if the treatments belong to the same group, while the variance of the difference for treatments that are members of different groups is the same for all such differences. From (2.1) and (2.2) it is easy to see that every treatment is equally replicated $r$ times, where $r=$ $c_{1}+s c_{2}+t c_{3}$. Finally, we consider only connected designs, so the rank of $\Omega^{-1}$ is $t$ (Rasch and Herrendörfer (1986, page 40)).

There are many important subclasses of GDDs. GDPBIBDs are the only GDDs that are proper and binary (Stidley (1988)). Treatments within the same group appear in $\lambda_{1}$ blocks together, while treatments in different groups appear together in $\lambda_{2}$ blocks. Let $k_{1}$ denote the common block size. It is easy to show that for GDPBIBDs, $c_{1}=r-\left(r-\lambda_{1}\right) / k_{1}, c_{2}=\left(\lambda_{2}-\right.$ $\left.\lambda_{1}\right) / k_{1}$ and $c_{3}=r / t-\lambda_{2} / k_{1}$.

If $c_{2}=0$ then the design is an EVBD or, equivalently, a Totally Balanced Design under Pearce's classification scheme. This class includes BIBDs and Extended Complete Block Designs, which were introduced by John (1963). Orthogonal designs are simply GDDs with $c_{2}=c_{3}=0$. Note that for any GDD with $c_{2}=0$ either (i) $m=1$ and $s=t$ or (ii) $s=1$ and $m=$ $t$. Both conditions imply that there is only a treatment or group factor, but not both. Without loss of generality when $c_{2}=0$, we refer to this factor as the treatment factor and assume $m=1$ and $s=t$.

The model that we consider is the additive mixed model such that

$$
Y=\mu I_{\mathrm{n}}+X_{\mathrm{M}^{\tau}}+X_{\mathrm{A}^{\alpha}}+X_{\mathrm{B}} \beta+\epsilon \text {, }
$$

where $Y$ is the $n$-vector of observations, $\mu$ is the mean, $I_{n}$ is an $n$-vector of ones, $\tau$ is the $m$-vector of group effects, $\alpha$ is the $t$-vector of treatment effects, $\beta$ is the $b$-vector of random block effects and $\epsilon$ is the vector of pure error terms. We assume that

$$
\begin{aligned}
& \text { (i) } \tau^{\prime} I_{m}=0 \\
& \text { (ii) } \alpha^{\prime} I_{m}^{\otimes I}=I_{m}^{\prime} \\
& \text { (iii) } \beta-N_{b}\left(O_{b}, \sigma_{b}^{2} I\right) \text { where } \sigma_{b}^{2} \geq 0 \\
& \text { (iv) } \epsilon-N_{n}\left(O_{n}, \sigma_{e}^{2} I\right) \text { where } \sigma_{e}^{2}>0
\end{aligned}
$$

and $(v) \quad \beta$ and $\epsilon$ are independent.

Thus, $E(Y)=\mu I_{n}+X_{M^{\tau}}+X_{A} \alpha$ and $\operatorname{cov}(Y)=\sigma_{b}^{2} X_{B} X_{B}^{\prime}+\sigma_{e}^{2} I_{n}$. The constraints placed on the fixed effects are made strictly for convenience and 
do not affect the outcome of the tests of hypotheses that we discuss later.

\section{DECOMPOSITION OF THE OBSERVATION SPACE}

We use the projective method (Christensen (1987)). Let $S$ be a (linear) subspace of the observation space, $\boldsymbol{R}^{n}$. Then the subspace of $\boldsymbol{R}^{\mathrm{n}}$ that is orthogonal to $S$ (with orthogonality defined with respect to the standard inner product) is denoted by $S^{\perp}$. If $T$ is a subspace orthogonal to $S$, then this relationship is written as $T \perp S$. Let $U$ be a subspace contained in $S$. Then $S \mid U$ denotes the subspace of $S$ that is orthogonal to $U$. The projection operator onto a subspace $S$ is denoted by $P_{S}$.

The observation space, $\boldsymbol{\Omega}^{n}$, is first decomposed into two orthogonal subspaces, the design space $V$ and the error space $V^{\perp}$. The design space, $V$ $=\operatorname{colsp}\left[I_{n}\left|X_{M}\right| X_{A} \mid X_{B}\right]$, which is the column space of the design matrices used in the model, regardless of the types of effects with which they are associated.

Let $A=\operatorname{colsp}\left(X_{\mathrm{A}}\right), M=\operatorname{colsp}\left(X_{M}\right)$ and $B=\operatorname{colsp}\left(X_{B}\right) . M$ and $B$ are the group and block spaces, respectively. Since the treatment effects are nested within the group effects, $A$ is actually the group and treatment space, with $A=M \oplus A \mid M$, where $A \mid M$ is the treatment adjusted for group space and $\oplus$ indicates the sum of orthogonal subspaces.

For orthogonal designs the design space is easily decomposed, since the relevant subspaces are orthogonal to one another. However, the subspaces are not orthogonal for GDDs. As a result, we consider three decompositions of the design space:

$$
\begin{aligned}
V & =1 \oplus B|1 \oplus V| B \\
V & =1 \oplus M|1 \oplus V| M \\
\text { and } \quad V & =1 \oplus A|M \oplus V|(A \mid(M \mid 1)),
\end{aligned}
$$

where $I$ denotes the mean space, $B \mid I$ and $M \mid I$ are the block and group spaces adjusted for the mean, and the spaces $V|B, V| M$ and $V \mid(A \mid(M \mid I))$ are the adjusted treatment and group space, adjusted block and treatment space and adjusted block and group space, respectively. The decomposition given in (3.1) is the intra-block decomposition, while the decompositions given in (3.2) and (3.3) are the intra-group and intra-treatment adjusted for groups decompositions. The sums of squares resulting from the intrablock, intra-group and intra-treatment adjusted for groups decompositions for nonorthogonal GDDs are given in the analysis of variance table in Table 1 .

The projection operators onto the spaces, $1, A, M, A|M, B, A| I, M \mid I$ and $B \mid I$ are easily obtained. For example, $P_{A}=X_{A}\left(X_{A}^{\prime} X_{A}\right)^{-1} X_{A}^{\prime}$. The projection operator onto the adjusted treatment and group space is given by

$$
\begin{aligned}
& P_{\mathrm{V} \mid \mathrm{B}}=\left(r / c_{1}\right)\left(P_{\mathrm{A} \mid \mathrm{M}}-P_{\mathrm{A} \mid \mathrm{M}} P_{\mathrm{B}}-P_{\mathrm{B}} P_{\mathrm{A} \mid \mathrm{M}}+P_{\mathrm{B}} P_{\mathrm{A} \mid \mathrm{M} P_{\mathrm{B}}}\right)+ \\
& {\left[r /\left(c_{1}+s c_{2}\right)\right]\left(P_{M \mid 1}-P_{M \mid 1} P_{B}-P_{B} P_{M \mid 1}+P_{B} P_{M \mid 1} P_{B}\right)}
\end{aligned}
$$

(Stidley (1988)). The expressions for the projection operators onto $V \mid A$, $V|M, V|(A \mid(M \mid 1))$ and $V^{\perp}$ are easily obtained from the expression for $V \mid B$.

The inter-block decompositions are also given in Table 1 . For these decompositions the block subspaces are further decomposed. If $c_{3} \neq 0$ then let 
If $s c_{2}+t c_{3} \neq 0$ then let

$$
\begin{aligned}
P_{\mathrm{M}^{\circ}} & =\left[r /\left(t c_{3}\right)\right] P_{\mathrm{B} \mid 1} P_{\mathrm{M}} P_{\mathrm{B} \mid I} \\
P_{\mathrm{EM}} & =P_{\mathrm{B} \mid I-P_{\mathrm{M}^{\circ}}} \\
\text { and } \quad P_{\mathrm{BM}} & =P_{\mathrm{V} \mid \mathrm{M}}-P_{\mathrm{EM}} .
\end{aligned}
$$

$$
\begin{aligned}
P_{\mathrm{A}^{\circ}} & =\left[r /\left(s c_{2}+t c_{3}\right)\right] P_{\mathrm{B}\left|I^{P} \mathrm{~A}\right| \mathrm{M}^{P} \mathrm{~B} \mid 1} \\
P_{\mathrm{EA}} & =P_{\mathrm{B} \mid 1-P_{\mathrm{A}^{\circ}}} \\
\text { and } P_{\mathrm{BA}} & =P_{\mathrm{V} \mid(\mathrm{A} \mid(\mathrm{M} \mid 1))-P_{\mathrm{EA}} .}
\end{aligned}
$$

It is easy to show that these matrices are projection operators onto subspaces of $B|1, V| M$ and $V \mid(A \mid(M \mid 1))$. Let $M^{\circ}, E M, B M, A^{\circ}, E A$ and $B A$ denote the subspaces onto which these operators project. The ranks of the matrices are equal to the corresponding degrees of freedom given in Table 1.

Note that for many GDDs, the ANOVA table can be simplified. For instance, the following relationships exist among the factor spaces (this result follows easily from Proposition 1 in the Appendix):

$$
M \mid 1 \perp B \text { if and only if } c_{3}=0
$$

and $(i i i)$

$$
A \mid M \perp B \text { if and only if } s C_{2}+t c_{3}=0
$$

Since the orthogonal case given in (iii) is trivial, we assume that at least one of the two scalars, $c_{2}$ and $c_{3}$, is nonzero.

The three major classes of GDPBIBDs are singular, semi-regular and regular GDPBIBDs. From the relationships among the factor spaces, it is easy to see that a GDPBIBD is singular if and only if $A \mid M \perp B$, while it is semi-regular if and only if $M \mid I \perp B$. For a regular GDPBIBD neither $M \mid I$ nor $A \mid M$ is orthogonal to $B$.

\section{DISTRIBUTION THEORY}

We are interested in testing for treatment, group and block effects. The specific hypotheses that we examine are:

(tg) Treatment and group hypotheses:

$$
\begin{aligned}
& \mathrm{H}_{0}: \tau_{i}=\alpha_{j}=0 \text { for all } i, j \text { vs } \\
& \mathrm{H}_{1}: \tau_{i} \neq 0 \text { or } \alpha_{j} \neq 0 \text { for at least one } i \text { or } j .
\end{aligned}
$$

(g) Group hypotheses:

$\mathrm{H}_{0}: \tau_{i}=0$ for all $i$ vs $H_{1}: \tau_{i} \neq 0$ for at least one $i$.

(t) Treatment hypotheses:

$$
\mathrm{H}_{0}: \alpha_{j}=0 \text { for all } j \text { vs } \mathrm{H}_{1}: \alpha_{j} \neq 0 \text { for at least one } j \text {. }
$$

(b) Block hypotheses:

$$
\mathrm{H}_{0}: \sigma_{\mathrm{b}}^{2}=0 \text { vs } \mathrm{H}_{1}: \sigma_{\mathrm{b}}^{2}>0 \text {. }
$$

We consider the various test statistics that can be constructed from the quadratic forms given in Table 1 . Let $P$ denote the projection operator onto a subspace and $\mathrm{X}^{2}(d, \delta)$ denote the chi-squared distribution 
with degrees of freedom $d$ and noncentrality parameter $\delta$. For the subspaces in Table 2, Y'PY is distributed as $\mathrm{w}_{1} \mathrm{X}^{2}\left(d, \mathrm{w}_{2} /\left(2 \mathrm{w}_{1}\right)\right)$ under the constraints listed in the table (see Proposition 2 in the Appendix). The parameters $w_{1}$ and $w_{2}$ are given in Table 2 , while the degrees of freedom $d$ are given in Table 1 . Note that some of the quadratic forms in Table 1 are not listed in Table 2. Standard distribution theory can be obtained for these sums of squares only under constraints that are so extreme the designs are trivial.

For the distribution theory for test statistics we consider three subclasses of GDDs, along with the general GDD. The subclasses are GDDs such that (i) $M \mid I \perp B$ (that is, $c_{3}=0$ ), (ii) $A \mid M \perp B$ (that is, $s c_{2}+t c_{3}$ $=0$ ) and (iii) $c_{2}=0$ (that is, $M \mid I=\phi$, the null space). By the results in Table 2 and independence of the appropriate quadratic forms, we can use the following statistics to test for treatment, group and block effects for these four classes of GDDs.

$$
\begin{aligned}
& {\left[Y^{\prime} P_{V \mid B} Y /(t-1)\right] /\left[Y^{\prime} P_{V^{\perp Y}} /(n-b-t+1)\right]} \\
& {\left[Y^{\prime} P_{M \mid I} Y /(m-1)\right] /\left[Y^{\prime} P_{V^{\perp}} Y /(n-b-t+1)\right]} \\
& {\left[Y^{\prime} P_{\mathrm{A} \mid \mathrm{M}^{Y}} /(t-\mathrm{m})\right] /\left[Y^{\prime} P_{\mathrm{V}^{\perp}} \mathrm{Y} /(n-b-t+1)\right]} \\
& {\left[Y^{\prime} P_{E A} Y /(b-t+m-1)\right] /\left[Y^{\prime} P_{V^{\perp}} Y /(n-b-t+1)\right]} \\
& {\left[Y^{\prime} P_{E M} Y /(b-m)\right] /\left[Y^{\prime} P_{V^{\perp Y}} /(n-b-t+1)\right]} \\
& {\left[Y^{\prime} P_{\mathrm{BA}} Y /(t-1)\right] /\left[Y^{\prime} P_{\mathrm{V}} \perp Y /(n-b-t+1)\right]} \\
& {\left[Y^{\prime} P_{M^{\circ}} Y /(m-1)\right] /\left[Y^{\prime} P_{E M} Y /(b-m)\right]} \\
& {\left[Y^{\prime} P_{\mathrm{A}} \circ Y /(t-m)\right] /\left[Y^{\prime} P_{E A} Y /(b-t+m-1)\right]}
\end{aligned}
$$

Let $\mathfrak{z}\left(d_{1}, d_{2}, \delta\right)$ denote the $F$-distribution with degrees of freedom $d_{1}$ and $d_{2}$ and noncentrality parameter $\delta$. Under the constraints given in Table 2 , test statistics (1) through (6) are distributed as $w \mathfrak{g}\left(d_{1}, d_{2}, \delta\right)$, where $w$ is the ratio of the numerator and denominator values for $w_{1}, d_{1}$ and $d_{2}$ are the numerator and denominator degrees of freedom, respectively, and $\delta^{2}$ is the numerator noncentrality parameter, $w_{2} /\left(2 w_{1}\right)$. Test statistics $(7)$ and $(8)$ are distributed as $w \mathfrak{g}\left(d_{1}, d_{2}, \delta\right)$ under the constraints in Table 2 and additional constraints that result in a zero value for the denominator noncentrality parameter. A derivation of these results is given in the Appendix. Recall that we are assuming that the design is nonorthogonal, so at least one of the scalars, $c_{2}$ and $c_{3}$, is nonzero.

Table 3 summarizes when these test statistics can be used to test the four hypotheses. We can test all of the hypotheses of interest for all three subclasses. Note the importance of the test statistics constructed from the inter-block decompositions. These decompositions are often ignored, but, obviously, can yield valuable information.

Note that when $c_{2}=0$, we have no group effect to test. Then statistics (1) and (8) can be used to test the same hypotheses. Since they are independent test statistics, they can be combined to obtain a possibly more powerful test (for example, by the method presented by Zelen (1957)) or the more powerful individual test may be used. With BIBDs, test statistic (1) always gives the more powerful test, but this result does not hold for all EVBDs (Stidley (1988)).

Calculations to obtain these test statistics can be obtained with any matrix programming language, such as Gauss (Aptech Systems (1988)). The intra-block, intra-group and intra-treatment statistics can be 
automatically obtained from standard analysis of variance packages, such as GLM in SAS (SAS (1985)), but the inter-block test statistics require further programming.

\section{EXAMPLE}

To illustrate these results we consider the example given in Table 4, which is a modification of an example given by Pearce (1965). The experiment consists of treating cherry trees with six fungicides, blocking for the treatment that was used on the trees during the previous year. Another blocking factor was also used, but as Pearce initially did, we omit this factor. We further modify the design by changing the block in which four observations occurred. The original two factor design is a GDD such that it is a generalization of an extended complete block design. The modification also has these characteristics, along with the property that $A \mid M$ is orthogonal to $B$. Thus, from Table 3 we see that we can test all four hypotheses. The calculations were done using Gauss (Aptech Sys tems (1988)). Table 5 gives the calculated values of each of the test statistics along with the p-values. For the treatment and group tests, the $p$-values are quite large, giving no evidence of a treatment or group effect. However, due to the small p-value for the block test, we conclude that $\sigma_{\mathrm{b}}^{2}>0$.

\section{CONCLUDING REMARKS}

The set of GDDs contains many important subsets of designs, such as BIBDs, GDPBIBDs and EVBDs. Although the class of designs is broad, $\$ 4$ gives a general distribution theory for testing treatment, group and block effects for the mixed model with random block effects. These test statistics are extensions of the intra-block and inter-block test statistics used in the analysis of BIBDs. The results presented in $\S 4$ and $\S 5$ show the importance of the inter-block test statistics in an analysis of nonorthogonal designs. However, while standard computer packages readily supply information on the intra-block type of test statistics, the interblock test statistics are not given. With the results presented here the inter-block test statistics for GDDs can be obtained by further programming.

\section{ACKNOWLEDGEMENTS}

Part of this research is contained in Chapter 4 of the author's Ph.D. dissertation written under the supervision of Professor Ronald M. Schrader. The author would like to thank Professor Schrader for helpful discussions and the editor and referees for their comments.

\section{REFERENCES}

Aptech Systems (1988). The Gauss System, Version 2. Kent, Washington. Christensen, R. (1987). Plane Answers to Complex Questions: The Theory of Linear Models. New York: Springer-Verlag.

Freeman, G.H. (1976). A cyclic method of constructing regular group divisible incomplete block designs. Biometrika, 63, 555-558.

Gupta, S.C., Jones, B. (1983). Equireplicate balanced block designs with unequal block sizes. Biometrika, 70, 443-440. 
Hedayat, A., Federer, W.T. (1974). Pairwise and variance balanced incomplete block designs. Ann. Inst. Stat. Math., 26, 331-338.

John, P.W.M. (1963). Extended complete block designs. Austral. J. Stat. , 5, 147-152.

Kageyama, S. (1981). Constructions of efficiency-balanced designs. Comm. Stat.-Theor. Meth., A10, 559-580.

Kageyama, S., Mohan, R.N. (1985). On a construction of group divisible partialiy balanced incomplete block designs. Sankyà, B47, 292-293.

Pearce, S.C. (1963). The use and classification of non-orthogonal designs. J. Roy. Stat. Soc. Ser. A., 126, 353-377.

Pearce, S.C. (1965). Biological Statistics: An Introduction. New York: McGraw-Hill.

Rasch, D., Herrendörfer, G. (1986). Experimental Design: Sample Size Determination and Block Designs. Dordrecht, Holland: D. Reidel.

SAS Institute, Inc. (1985). SAS User's Guide: Statistics, Version 5 Edition. Cary, North Carolina: SAS Institute, Inc.

Sinha, K. (1987). A method of construction of regular group divisible designs. Biometrika, 74, 443-444.

Stidley, C.A. (1988). The analysis of mixed and random effects models for nonorthogonal designs. Ph.D. thesis, Department of Mathematics and Statistics, University of New Mexico.

Tocher, K.D. (1952). The design and analysis of block experiments. J. Roy. Stat. Soc. Ser. B., 14, 45-100.

Tyagi, B.N. (1979). On a class of variance balanced block designs. J. Stat. Plan. Inf., 3, 333-336.

Zelen, M. (1957). The analysis of incomplete block designs. J. Amer. Stat. Assn., 52, 204-217.

\section{APPENDIX}

The distribution theory for the quadratic forms and the test statistics is derived. First, the following results are needed.

Proposition 1.

$$
\begin{aligned}
& P_{\mathrm{A}} P_{\mathrm{B}} P_{\mathrm{A}}=\left[\left(s c_{2}+t c_{3}\right) / r\right] P_{\mathrm{A} \mid \mathrm{M}}+\left(t c_{3} / r\right) P_{\mathrm{M} \mid 1}+P_{1} \\
& P_{\mathrm{M} \mid 1} P_{\mathrm{B}} P_{\mathrm{M} \mid 1}=\left(t c_{3} / r\right) P_{\mathrm{M} \mid 1}
\end{aligned}
$$

and $\quad P_{\mathrm{A} \mid \mathrm{M}} P_{\mathrm{B}} P_{\mathrm{A} \mid \mathrm{M}}=\left[\left(s c_{2}+t c_{3}\right) / r\right] P_{\mathrm{A} \mid \mathrm{M}}$.

Proof.

$$
P_{\mathrm{A}} P_{\mathrm{B}} P_{\mathrm{A}}=\left(1 / r^{2}\right) X_{\mathrm{A}} N K^{-1} N^{\prime} X_{\mathrm{A}}^{\prime} \text {. }
$$

Note that for equireplicate designs

$$
\Omega^{-1}=r I_{t}-N K^{-1} N^{\prime}+(r / t) J_{t}
$$

while for GDDs

$$
\Omega^{-1}=c_{1} I_{t}+c_{2} I_{m} \otimes J_{s}+c_{3} J_{t} .
$$

Since $r=c_{1}+s c_{2}+t c_{3}$, from the above equations we obtain

$$
N K^{-1} N^{\prime}=\left(s c_{2}+t c_{3}\right) I_{t}-c_{2} I_{m} \otimes J_{s}+\left[\left(c_{1}+s c_{2}\right) / t\right] J_{t} \text {. }
$$

The result for $P_{A} P_{B} P_{A}$ is obtained by substituting this expression into $A .1$ and simplifying.

Since $M$ is contained in $A, \quad P_{M \mid I} P_{B} P_{M \mid I}=P_{M \mid 1} P_{A} P_{B} P_{A} P_{M} \mid I=$ $\left(t c_{3} / r\right) P_{M \mid I}$. The result for $P_{A \mid M} P_{B} P_{A \mid M}$ follows similarly. 
The following corollary is obtained from repeated applications of Proposition 1.

Corollary 1. If $k=k_{1} I_{\mathrm{b}}$ then let $M_{1}=\left[\left(k_{1} t c_{3} / r\right) \sigma_{\mathrm{b}}^{2}+\sigma_{\mathrm{e}}^{2}\right]^{-1} P_{\mathrm{M} \mid 1} \operatorname{cov}(Y)$ and $M_{2}=\left\{\left[k_{1}\left(s c_{2}+t c_{3}\right) / r\right] \sigma_{\mathrm{b}}^{2}+\sigma_{\mathrm{e}}^{2}\right\}^{-1} P_{\mathrm{A} \mid \mathrm{M}} \operatorname{cov}(Y)$, while if $k=k_{1} I_{\mathrm{b}}$ and $c_{2}=0$, then let $M_{3}=\left[\left(k_{1} c_{1} / r\right) \sigma_{\mathrm{b}}^{2}+\sigma_{\mathrm{e}}^{2}\right]^{-1} P_{\mathrm{BA}} \operatorname{cov}(Y)$. Then the matrices $M_{1}, M_{2}$ and $M_{3}$ are idempotent.

The following results give the distribution theory presented in $\$ 4$. Proposition 2. Let $S$ be one of the subspaces listed in Table 2. Then, subject to the conditions given in Table 2, Y'P $Y$ is distributed as $w_{1} X^{2}\left(d, w_{2} /\left(2 w_{1}\right)\right)$, where $w_{1}$ and $w_{2}$ are given in Table 2 and $d$ is the degrees of freedom given in Table 1 .

Proof. Let $P$ be the projection operator onto one of the subspaces. Then

$$
P \operatorname{cov}(Y)=\sigma_{\mathrm{b}}^{2} P X_{\mathrm{B}} X_{\mathrm{B}}^{\prime}+\sigma_{\mathrm{e}}^{2} P \text {. }
$$

Under the conditions given in Table 2, it can be shown that either

$$
\text { (i) } P \operatorname{cov}(Y)=w_{1} P
$$

or (ii) Corollary 1 holds.

Thus, $P \operatorname{cov}(Y) / w_{1}$ is idempotent, so $Y^{\prime} P Y$ is distributed as $w_{1} X^{2}(d, \delta)$, where $\delta=E(Y)$ ' $P E(Y) /\left(2 w_{1}\right)$. The derivation of the expressions for the noncentrality parameter uses the expression for $N K^{-1} N^{\prime}$ given in (A.2) and the relationship between $X_{A}$ and $X_{M}$, which is, $X_{M}=X_{A}\left(I_{m} \otimes I_{s}\right)$.

Corollary 2. Let $F$ be one of the eight listed test statistics. Consider the conditions for the numerator and denominator quadratic forms given in Table 2. For test statistic (7) add the constraint that $s c_{2}+t c_{3}=0$, while for (8) consider two situations. First, add the constraint that $c_{3}$ $=0$, while for the second case let the additional constraint be that $c_{2}=$ 0 . Let $w$ be the ratio of the numerator $w_{1}$ to the denominator $w_{1}$. Then, under these conditions, $F$ is distributed as $w \mathfrak{z}\left(d_{1}, d_{2}, \delta\right)$.

Proof. Let $P$ denote the projection operator onto the subspace corresponding to one of the numerator sums of squares. Then the column space of $P$ is contained in $V$ and $P \operatorname{cov}(Y) P_{V^{\perp}}=0$. If $k=k_{1} l$ and $c_{3} \neq 0$, then $M^{\circ}$ is orthogonal to EM. Then

$$
P_{\mathrm{M}} \circ \operatorname{cov}(Y) P_{\mathrm{EM}}=\left(k_{1} \sigma_{\mathrm{b}}^{2}+\sigma_{\mathrm{e}}^{2}\right) P_{\mathrm{M}^{\circ}} P_{\mathrm{EM}}=0
$$

A similar result holds for $P_{A^{\circ}}$ and $P_{E A}$. Thus, the numerator and denominator quadratic forms are independent. The result then follows from Proposition 2. 
Table 1

Analysis of Variance Table for Nonorthogonal Group Divisible Designs

Table 1.1 Intra-block decomposition

\begin{tabular}{|c|c|c|}
\hline Source & SS & $d f$ \\
\hline Blocks, ignoring treatments and groups & $Y^{\prime} P_{B \mid I^{Y}}$ & $b-1$ \\
\hline \multicolumn{3}{|l|}{ Inter-block decompositions: } \\
\hline \multirow{2}{*}{$\begin{aligned} \text { Decomposition 1: } & \text { Group component } \\
& \text { Inter-block/group error }\end{aligned}$} & $Y^{\prime} P_{M^{\circ}} Y$ & $m-1$ \\
\hline & $Y^{\prime} P_{E M} Y$ & $b-m$ \\
\hline \multicolumn{3}{|l|}{ Decomposition 2: Treatments nested within } \\
\hline groups component & $Y^{\prime} P_{A} \circ Y$ & $t-m$ \\
\hline Inter-block/treatment error & $Y^{\prime} P_{E A^{Y}}{ }^{Y}$ & $D-t+m-I$ \\
\hline Treatments \& groups, eliminating blocks & $Y^{\prime} P_{V \mid B} Y$ & $t-1$ \\
\hline Intra-block error & $Y^{\prime} P_{V^{\perp Y}}$ & $n-b-t+1$ \\
\hline Total, adjusted for the mean & $Y^{\prime} P_{I} \perp Y$ & $n-1$ \\
\hline
\end{tabular}

Table 1.2 Intra-group decomposition

\begin{tabular}{lcc}
\multicolumn{1}{c}{ Source } & SS & df \\
\hline Groups, ignoring treatments and blocks & $Y^{\prime} P_{M} \mid I^{Y}$ & $m-1$ \\
Treatments \& blocks, eliminating groups & $Y^{\prime} P_{V \mid M} Y$ & $b+t-m-1$ \\
Inter-block decomposition: Block component & $Y^{\prime} P_{B M} Y$ & $t-1$ \\
& $Y^{\prime} P_{E M} Y$ & $b-m$ \\
Intra-block error & $Y^{\prime} P_{V^{\perp}}$ & $n-b-t+1$ \\
\hline Total, adjusted for the mean & $Y^{\prime} P_{1} \perp Y$ & $n-1$
\end{tabular}

Table 1.3 Intra-treatment adjusted for group decomposition

\begin{tabular}{|c|c|c|}
\hline Source & SS & $d f$ \\
\hline Treatments, ignoring groups and blocks & $Y^{\prime} P_{A} \mid M^{Y}$ & $t-m$ \\
\hline \multicolumn{3}{|l|}{ Groups \& blocks, eliminating treatments nested } \\
\hline within groups & $Y^{\prime} P_{V} \mid(A \mid(M \mid I))^{Y}$ & $b+m-2$ \\
\hline Inter-block decomposition: Block component & $Y^{\prime} P_{B A} Y$ & $t-1$ \\
\hline Inter-block/treatment error & $Y^{\prime} P_{E A} Y$ & $b-t+m-1$ \\
\hline Intra-block error & $Y^{\prime} P_{V^{\perp Y}}$ & $n-b-t+1$ \\
\hline 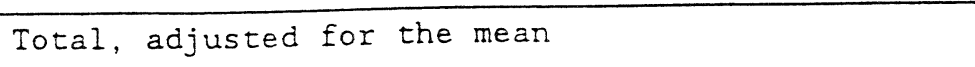 & $Y^{\prime} P_{1} \perp Y$ & $n-1$ \\
\hline
\end{tabular}


Table 2

Distribution Parameters and Constraints

Sub -

\begin{tabular}{|c|c|c|c|}
\hline space & $w_{1}$ & $w_{2}$ & Constraints \\
\hline$B \mid 1$ & $k_{1} \sigma_{b}^{2}+\sigma_{e}^{2}$ & $s t c_{3} \tau^{\prime} \tau+\left(s c_{2}+t c_{3}\right) \alpha^{\prime} \alpha$ & $k=k_{1} I_{b}$ \\
\hline$M \mid 1$ & $\left(k_{1} t c_{3} / r\right) \sigma_{\mathrm{b}}^{2}+\sigma_{\mathrm{e}}^{2}$ & $r S T^{\prime} T$ & $k=k_{1} I_{b}$ \\
\hline$A \mid M$ & {$\left[k_{1}\left(s c_{2}+\tau c_{3}\right) / r\right] \sigma_{b}^{2}+\sigma_{e}^{2}$} & $r \alpha^{\prime} \alpha$ & $k=k_{1} I_{b}$ \\
\hline$V \mid B$ & $\sigma_{e}^{2}$ & $s\left(c_{1}+s c_{2}\right) \tau^{\prime} \tau+c_{1} \alpha^{\prime} \alpha$ & none \\
\hline$M^{\circ}$ & $k_{1} \sigma_{b}^{2}+\sigma_{e}^{2}$ & $s t c_{3} \tau^{\prime} \tau$ & $k=k_{1} I_{b} ; c_{3}=0$ \\
\hline EM & $k_{1} \sigma_{b}^{2}+\sigma_{e}^{2}$ & $\left(s c_{2}+t c_{3}\right) \alpha^{\prime} \alpha$ & $k=k_{1} I_{b} ; c_{3} \neq 0$ \\
\hline$A^{\circ}$ & $k_{1} \sigma_{\mathrm{b}}^{2}+\sigma_{\mathrm{e}}^{2}$ & $\left(s c_{2}+t c_{3}\right) \alpha^{\prime} \alpha$ & $=k_{1} I_{b} ; s c_{2} \nLeftarrow-t c_{3}$ \\
\hline$E A$ & $k_{1} \sigma_{\mathrm{b}}^{2}+\sigma_{\mathrm{e}}^{2}$ & $\operatorname{stc}_{3} \tau^{\prime} \tau$ & $=k_{1} I_{b} ; s c_{2} \approx-t c_{3}$ \\
\hline $\mathrm{BA}$ & $\left(k_{1} c_{1} / r\right) \sigma_{\mathrm{b}}^{2}+\sigma_{\mathrm{e}}^{2}$ & 0 & $k=k_{1} I_{b} ; c_{2}=0$ \\
\hline $\mathrm{V}^{\perp}$ & $\sigma_{e}^{2}$ & 0 & none \\
\hline
\end{tabular}

Table 3

Summary of Tests of Fixed and Random Effects

for Subclasses of Group Divisible Designs

Subclass of Group Divisible Designs

\begin{tabular}{c|cccc} 
Hypothesis & general & $\begin{array}{c}M \mid 1 \perp B \\
\left(c_{3}=0\right)\end{array}$ & $\begin{array}{c}A \mid M \perp B \\
\left(s c_{2}=-t c_{3}\right)\end{array}$ & $\begin{array}{c}c_{2}=0 \\
\left(M^{2} \mid 1=\phi\right)\end{array}$ \\
\hline$t \& g$ & 1 & 1 & 1 & \\
$g$ & & $2^{\dagger}$ & $7^{\dagger}$ & $1,88^{\dagger}$ \\
$t$ & & $8^{\dagger}$ & $3^{\dagger}$ & $4^{\dagger}, 6^{\dagger}$
\end{tabular}

Additional constraint required: design is proper.

* Additional constraints required: design is proper and $b \neq t$.

* Test statistics 1 and 8 are independent for this subclass.

Note: Any number within the table refers to one of the eight test statistics. For the appropriate design, this statistic can be used to test the corresponding hypothesis. 
Table 4

Example

Cherry crop yield (pounds)

Fungicide

\begin{tabular}{c|cccccc} 
Blocks & A & B & C & D & $E$ & F \\
\hline 1 & 16.7 & 18.6 & 13.1 & 8.9 & $12.8,13.6$ & $12.5,10.9$ \\
2 & 7.2 & 8.1 & 7.6 & 13.8 & $9.9,9.4$ & $1.2,15.9$ \\
3 & $12.3,10.9$ & $10.1,5.4$ & 16.3 & 17.9 & 15.3 & 7.8 \\
4 & 11.7 & 15.3 & $13.5,19.3$ & $14.6,15.5$ & 12.2 & 9.0 \\
5 & 15.0 & 7.3 & $7.5,8.9$ & $9.8,12.7$ & 10.0 & 10.9 \\
6 & $9.2,10.4$ & $13.3,16.7$ & 15.7 & 9.6 & 17.4 & 10.3
\end{tabular}

Table 5

\begin{tabular}{crrrrrrr}
\multicolumn{7}{c}{ Summary of Hypothesis Testing for Example } \\
Hypothesis & \multicolumn{2}{c}{$\begin{array}{c}\text { numerator } \\
\text { SS }\end{array}$} & df & \multicolumn{2}{c}{ denominator } \\
\hline$t g$ & 49.33 & 5 & 478.7 & 37 & 0.76 & 0.58 \\
$g$ & 11.18 & 2 & 127.5 & 3 & 0.13 & 0.88 \\
$t$ & 30.70 & 3 & 478.7 & 37 & 0.79 & 0.51 \\
$\mathrm{~b}$ & 127.50 & 3 & 478.7 & 37 & 3.29 & 0.03
\end{tabular}

